\title{
Adaptive PDF Speed Control for Motion Control
}

\author{
Laszlo Szamel* $^{*}$ \\ * Budapest University of Technology and Economics/Department of Electric Power Engineering, Budapest, Hungary, \\ e-mail: szamel.laszlo@vet.bme.hu
}

\begin{abstract}
This paper proposes two model reference adaptive PDF speed control methods. Following from the structure of model reference parameter adaptive control it makes it easier to reach overshootless. The approaching block diagram of the model reference signal adaptive control can be seen as an extended version of the PDF controller.
\end{abstract}

Keywords-Adaptive control, Adjustable speed drive, Energy storage, Motion control, Servo-drive, Switched reluctance drive, Variable speed drive.

\section{INTRODUCTION}

Generally the controller of the speed loop in motion control systems is of PI-type. The integrator of the controller eliminates the error caused by the step change in load. The setting of speed controller is difficult as the closed-loop transfer functions are not identical to step changes in the load, as well as the reference signal. It seems to be preferable to use PF-type controller (proportional gain in a separate feedback) instead of the traditional PI one. It means relatively simple solution [1], [2], [3]. Phelan named this structure "Pseudo-derivative feedback" (PDF) control [4].

In motion control systems there is robustness against parameter changes and disturbance rejection of main interest. The model reference adaptive control has the following features:

- it enables the compliance of the system with varying operational conditions possible and ensures the behavior of the controlled system according to the prescribed reference model;

- it means such a special type of adaptive systems which results in nonlinear control systems. This is the reason why the analytical analysis is completed by the Lyapunov stability criterion or by hyperstability principle;

- its design and application are closely related to the using of computer methods;

- simple implementation of the control algorithm.

In this paper the application of two model reference adaptive PF control methods to servo drives with switched reluctance motors are presented. Following from the PFtype structure model reference parameter adaptive control was developed to provide constant loop gain in speed control loop with changing gain (moment of inertia and/or torque factor) it makes it easier to reach overshootless as well as fast speed changing compensation caused by jump in load. Model reference signal adaptive control is also used to provide constant loop gain in speed control loop with changing parameters exposed to a significant load.
The approaching block diagram of the adaptive control can be seen as an extended version of the PF controller.

\section{Speed CONTOl of Servo Drives}

A model reference adaptive control is used for the speed control. Such an adaptive control has been succesfully elaborated by using a suitable chosen Lyapunov function to compensate the gain of the speed control loop [5], [6].

\section{A. Model Reference Parameter Adaptive Control}

The adaptive control of servo-drives with a cascade arrangement is most effective when it is applied in the inner loop containing the effect of variable parameters directly, i.e. the inertia $\left(J_{m}\right)$ and/or torque factor $\left(k_{m}\right)$. The speed control implemented by PI controller is of cascade arrangement in fact as it contains an inner, proportional feedback loop (PF controller, Fig.1.). A onestorage proportional element can describe this inner loop neglecting the time constant of closed current control loop. By this our adaptation algorithm is simplest.

Relation between accelerating current determining dynamic torque and angular velocity can be given by the following transfer function:

$$
Y_{\omega,\left(i-i_{t}\right)}(s)=\frac{A_{i}}{s},
$$

where $A_{i}=\frac{k_{m}}{J_{m}}$.

Arrangement of control circuit can be seen in the following figure:

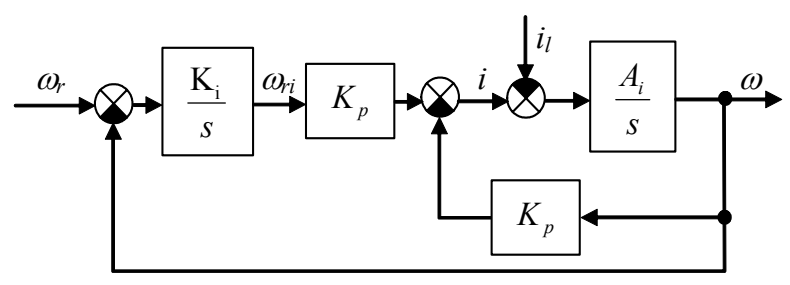

Fig. 1. Block scheme of parameter adaptive PF speed control

where
$\omega \quad$ is the speed,
$\omega_{r} \quad$ is the speed reference signal,
$i \quad$ is the current of the motor,
$i_{l} \quad$ is the current equivalent to the load- torque. 
Section determined by the transfer function $Y_{\omega,\left(i-i_{t}\right)}(s)$ is fed back by a proportional member of gain $K_{P}$. The task is to change the gain $K_{P}$ in such a way that the product $A_{i} K_{P}$ should remain constant despite the changing of parameter $A_{i}$.

Transfer factor of the inner closed loop is given by the reciprocal $\left(1 / K_{P}\right)$ of feedback member that is not constant because of the change in torque factor. Consequently loop gain of the outer speed control loop would change as well. In order to get a one-storage element with unity transfer factor we have to insert a member with gain $K_{P}$ between the integrator of the PF controller and the reference signal of the inner loop. Firstorder reference model with time constant $T_{m}$ gets sum of the input signals of above member $\left(\omega_{r i}\right)$ and the signal $\omega_{l m}$ compensating the load effect for the model. So dynamics of reference model can be described by the following differential equation:

$$
\dot{\omega}_{m} T_{m}+\omega_{m}=\omega_{r i}+\omega_{l m} .
$$

Dividing (2) with $T_{m}$ and applying designation $q_{m}=1 / T_{m}$, we get the following equation.

$$
\dot{\omega}_{m}+q_{m} \omega_{m}=q_{m}\left(\omega_{r i}+\omega_{l m}\right) .
$$

The differential equation of the first-order controlled section is as follows:

$$
\dot{\omega}+\left(K_{p} A_{i}\right) \omega=\left(K_{p} A_{i}\right) \omega_{r i}-A_{i} i_{l} .
$$

Factor $K_{p}$ can be described as the sum of $K_{p 0}$ determined by mean $A_{i}$ and $\Delta K_{p}$ accomplished by the adaptation algorithm. So:

$$
K_{p} A_{i}=\left(K_{p 0}+\Delta K_{p}\right) A_{i}=q+\Delta q,
$$

where $K_{P 0}$, and $q$ are constant.

In this case we assume that the change of $A_{i}$ is slow from the viewpoint of adaptation and therefore the effect of this change can be neglected.

Substituting (5) into (4) we get:

$$
\dot{\omega}+(q+\Delta q) \omega=(q+\Delta q) \omega_{r i}-A_{i} i_{l} .
$$

By using (3) and (6) and substituting expression of model error $\varepsilon=\omega_{m}-\omega$ the dynamic equation will be:

$$
\dot{\varepsilon}=-q_{m} \varepsilon+x \omega-x \omega_{r i}+q_{m} \omega_{l m}+A_{i} i_{l},
$$

where $x=(q+\Delta q)-q_{m}$.

Dynamic of model error should by asymptotically stable to follow the system with model proposed. For determination of $\Delta q$ the following Lyapunov function should be composed:

$$
V=\frac{1}{2}\left(\varepsilon^{2}+\beta x^{2}\right),
$$

When choosing the Lyapunov function both purposes, i.e. the termination of the model error $\left(\varepsilon=\omega_{m}-\omega\right)$ and loop gain deviation have been taken account.

The time derivative of the Lyapunov function is:

$$
\dot{V}=\varepsilon \dot{\varepsilon}+\beta x \dot{x} .
$$

Substituting (7) into (9) the following equation is valid:

$$
\begin{aligned}
& \dot{V}=-q_{m} \varepsilon^{2}+\varepsilon \times \omega-\varepsilon \times \omega_{r i}+ \\
& \varepsilon\left(q_{m} \omega_{l m}+A_{i} i_{l}\right)+\beta x \dot{x} .
\end{aligned}
$$

If

$$
\varepsilon \times \omega-\varepsilon \times \omega_{r i}+\beta x \dot{x}=0,
$$

that is

$$
\dot{x}=\varepsilon\left(\omega_{r i}-\omega\right) / \beta,
$$

and

$$
\varepsilon\left(q_{m} \omega_{l m}+A_{i} i_{l}\right)<0
$$

then

$$
\dot{V}<-q_{m} \varepsilon^{2} .
$$

The above equation is a negative definite function that shows the asymptotic stability of the error dynamic (7). By using (5), (7) and (12) the following adaptation algorithm is true:

$$
\Delta \dot{K}_{p}=\gamma \varepsilon\left(\omega_{r i}-\omega\right),
$$

where $\gamma$ may be an arbitrary positive number. The inequality (13) shows how we have to change the signal $\omega_{l m}$ representing the load of model.

\section{If}

$$
\varepsilon>0, \text { then } \omega_{l m}<-\frac{\left|i_{l}\right|_{\max } A_{i}}{q_{m}},
$$

respectively if

$$
\varepsilon<0, \text { then } \omega_{l m}>\frac{\left|i_{l}\right|_{\max } A_{i}}{q_{m}} .
$$

\section{B. Model Reference Signal Adaptive Control}

The controlled loop has been approximated by an integral element. Time constant of the closed current control loop has been neglected. The control consists of a P-element with the gain $K_{p}$. Input of P-element contains not only the control error signal but an adaptation signal as well $(g)$. Applying the signal adaptation control, a P type controller with $K_{p}$ gain can ensure zero speed error as the adaptation signal can produce a current reference signal to compensate the loading current at zero speed error.

where $\beta$ is a positive number. 


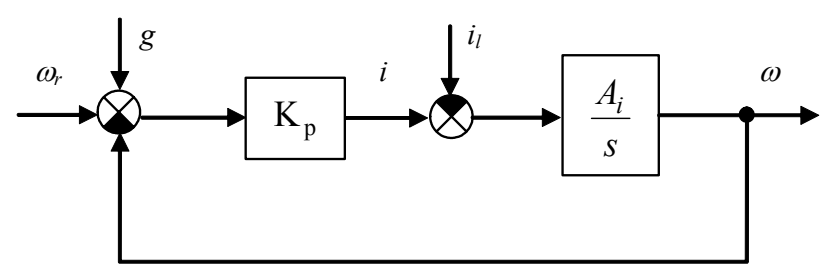

Fig. 2. Initial block scheme of the signal adaptive speed control

Regarding the block diagram of control loop following differential equation is valid for the closed loop:

$$
\dot{\omega}+A_{i} K_{p} \omega=A_{i} K_{p}\left(\omega_{r}+g\right)-A_{i} i_{l} .
$$

The feature of the closed speed control loop has been taken into consideration by a parallel control model to be expressed by a first order proportional element. The differential equation of the first order system is:

$$
\dot{\omega}_{m}+q_{m} \omega_{m}=q_{m} \omega_{r},
$$

where index $\mathrm{m}$ refers to the model and $q_{m}$ is the reciprocal of model time constant.

Using (17) and (18) and introducing the expression $\varepsilon=\omega_{m}-\omega$ for model error, the dynamic equation for the model error is as follows:

$$
\dot{\varepsilon}+q_{m} \varepsilon=\left(q_{m}-A_{i} K_{p}\right)\left(\omega_{r}-\omega\right)+A_{i}\left(i_{l}-K_{p} g\right) .
$$

The adaptation signal $g(t)$ can be written in the following form:

$$
g(t)=g_{1}(\mathrm{t})\left(\omega_{r}-\omega\right)+g_{2}(t) .
$$

Substituting (20) for (19):

$$
\dot{\varepsilon}=-q_{m} \varepsilon+b_{1}\left(\omega_{r}-\omega\right)+b_{2},
$$

where:

$$
\begin{aligned}
& b_{1}=q_{m}-A_{i} K_{p}\left(1+g_{1}(t)\right), \\
& b_{2}=A_{i}\left(i_{l}-K_{p} g_{2}(t)\right) .
\end{aligned}
$$

Let us compose the following Lyapunov function to produce the signal $g_{1}(t)$ and $g_{2}(t)$ :

$$
V=\frac{1}{2} \varepsilon^{2}+\frac{1}{2}\left(\beta_{1} b_{1}^{2}+\beta_{2} b_{2}^{2}\right),
$$

where $\beta_{1}$ and $\beta_{2}$ are positive constants.

The Lyapunov function has been chosen in such a way that model error should be decreased asymptotically and gain of speed control loop and load should be compensated. In this case we have to assume that change in loop gain and in load is smaller than the speed of the adaptation.

Time-derivation of the Lyapunov function is:

$$
\dot{V}=\varepsilon \dot{\varepsilon}+\beta_{1} b_{1} \dot{b}_{1}+\beta_{2} b_{2} \dot{b}_{2} .
$$

Substituting (21) for (23):

$$
\begin{aligned}
\dot{V}= & -q_{m} \varepsilon^{2}+\left(\omega_{r}-\omega\right) b_{1} \varepsilon+b_{2} \varepsilon \\
& +\beta_{1} b_{1} \dot{b}_{1}+\beta_{2} b_{2} \dot{b}_{2} .
\end{aligned}
$$

If

$$
\dot{b}_{1}=-\left(\omega_{r}-\omega\right) \varepsilon / \beta_{1},
$$

and

$$
\dot{b}_{2}=-\varepsilon / \beta_{2},
$$

then

$$
\dot{V}=-q_{m} \varepsilon^{2},
$$

and it ensures asymptotical stability of the model error. On the basis of (21), (25), (26) and by assuming that variation of $A_{i}$ can be neglected compared to the speed of adaptation the following adaptation algorithm is valid:

$$
\begin{gathered}
\dot{g}_{1}(t)=\gamma_{1} \varepsilon\left(\omega_{r}-\omega\right), \\
\dot{\mathrm{g}}_{2}(\mathrm{t})=\gamma_{2} \varepsilon,
\end{gathered}
$$

where $\gamma_{1}$ and $\gamma_{2}$ are positive constants, the free parameters of adaptation. Taking relations (20), (28), (29) into consideration the following equation comes true:

$$
g(t)=\gamma_{1}\left(\omega_{r}-\omega\right) \int \varepsilon\left(\omega_{r}-\omega\right) d t+\gamma_{2} \int \varepsilon d t .
$$

A block diagram of the control circuit introducing adaptation signal $g(t)$ and assuming that $g_{1}(t)$ is constant can be seen in Fig. 3. Taking the structure of control: it contains two parts. In the first part the reference signal is led through a first order system and a PI controller with variable gain and integration time. The second one is a differentiating filter which takes effect only on changing of reference signal. The gain and differentiation time are also changing. The adaptation gain factor $\gamma_{2}$ gives the reciprocal of integrating time constant of controller type PI, assuming $g_{1}(t)=0$. To fulfill the constant integrating time constant it is preferable to substitute $\gamma_{2}$ by $\gamma_{2}\left(1+g_{1}(t)\right)$. In such a way the neglect of time constant of current control loop can be compensated.

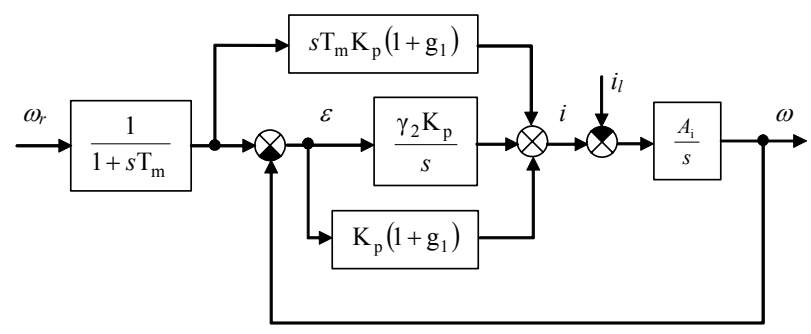

Fig. 3. Block scheme of the signal adaptive speed control with adaptation signal

The approaching block diagram of the adaptive control can be seen as extended version of the PI controller, so $\gamma_{2}$, one of the adaptation factors (which is the free parameter of the adaptive control) is given. Contraction of model-filtered reference signal and the PI controller can be transformed into a so-called PF controller when 
integration time of PI controller equals to the time constant of the model (Fig.4.)

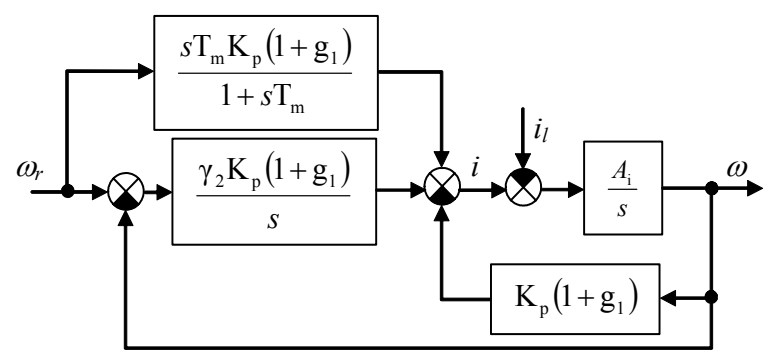

Fig. 4. Block scheme of the signal adaptive PF speed control (extended version)

The basic structure of the signal adaptive speed controller is also PF type which on the one hand provides overshootless with its structure. Moreover, it ensures fast compensation of speed variation caused by a jump in motor load.

The transfer-function between $\omega$ and $\omega_{r}$ is as follows:

$$
\frac{\omega}{\omega_{r}}=\frac{1}{1+s T_{m}} \frac{1+s T_{m}+s^{2} \frac{T_{m}}{\gamma_{2}}}{1+s \frac{1}{\gamma_{2}}+s^{2} \frac{1}{A_{i} \gamma_{2} K_{p}\left(1+g_{1}\right)}} .
$$

Assuming that the adaptation signal $g_{1}(t)$ is constant (at the end of the adaptation), the following equation is valid:

$$
\left(1+g_{1}\right)=\frac{\gamma_{2}}{A_{i} K_{P}}
$$

Substituting (32) into (31):

$$
\frac{\omega}{\omega_{r}}=\frac{1}{1+s T_{m}} \frac{1+s T_{m}+s^{2} \frac{T_{m}}{\gamma_{2}}}{1+s \frac{1}{\gamma_{2}}+s^{2} \frac{1}{\gamma_{2}^{2}}} .
$$

Choosing $T_{m}=\frac{1}{\gamma_{2}}$, the transfer function is as follows:

$$
\frac{\omega}{\omega_{r}}=\frac{1}{1+s T_{m}} .
$$

So the system follows the model without time delay.

This control has been tested by a simulation program developed in our Department. First the adaptation has been examined without current limitation and load in order to take into consideration only the non-linearity of the motor and the adaptation. In the interest of the adaptation stability the speed of change of the adaptation signal $g_{1}$ has to be limited. The signal $g_{1}$ can results in the significant oscillations without limitations as the change of the signal is possible in discrete times.
The current limitation can results in further problems. This limitation hinders the tracking of the model, so the effect of the above signal $g_{1}$ will be too large or it can change in the reverse direction. To eliminate the above problem signal $g_{1}$ is not to be changed in the period of the current limitation.

\section{DRIVE SYSTEM}

Block scheme of the examined drive system with SRM (switched reluctance motor) [7], [8] is shown in Fig. 5. This work is a part of the investigation of an HTS (high temperature superconductor) energy storage flywheel system [9]. The main advantages of this motor type are the following:

- no need for an extra generator;

- relatively big torque can be achieved, thus easier to pass critical speeds;

- no iron losses at stand-by.

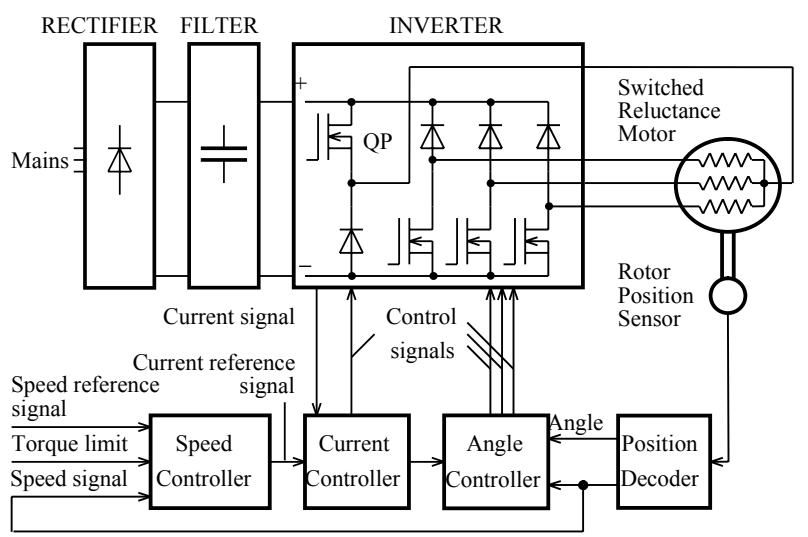

Fig. 5. Block scheme of drive system

It follows from the operational principle of SRM that its phase windings are to be excited at a well determined angle of the rotor position in an appropriate order. This is why a Rotor Position Sensor is to be mounted on the shaft of the motor. In our case the position sensor is a resolver. It can be calculated from the teeth numbers $(6 / 8)$ that the phase switchings have to follow each other by 15 degree.

The supply unit consists of three main blocks, namely the RECTIFIER, the FILTER and the INVERTER. The inverter is a pulsed width modulated (PWM) one, marked by QP in the figure and it contains a one-one switching transistor per phase and a brake chopper, not shown in the figure. The common point of phase windings is supplied by the PWM inverter. It is of autonomous operation and has an inner current control loop. The other ends of phase windings are connected to the phase switching transistors.

\section{Simulation Results}

In Fig. 6 and Fig. 7 two of many executed simulations are shown. Fig. 6 shows the run-up with model reference parameter adaptive control (15), (16), while Fig. 7 with model reference signal adaptive control (30). and in both cases with turn-on and turn-off angles depending on the speed and current reference and with current reference compensation [10]. 

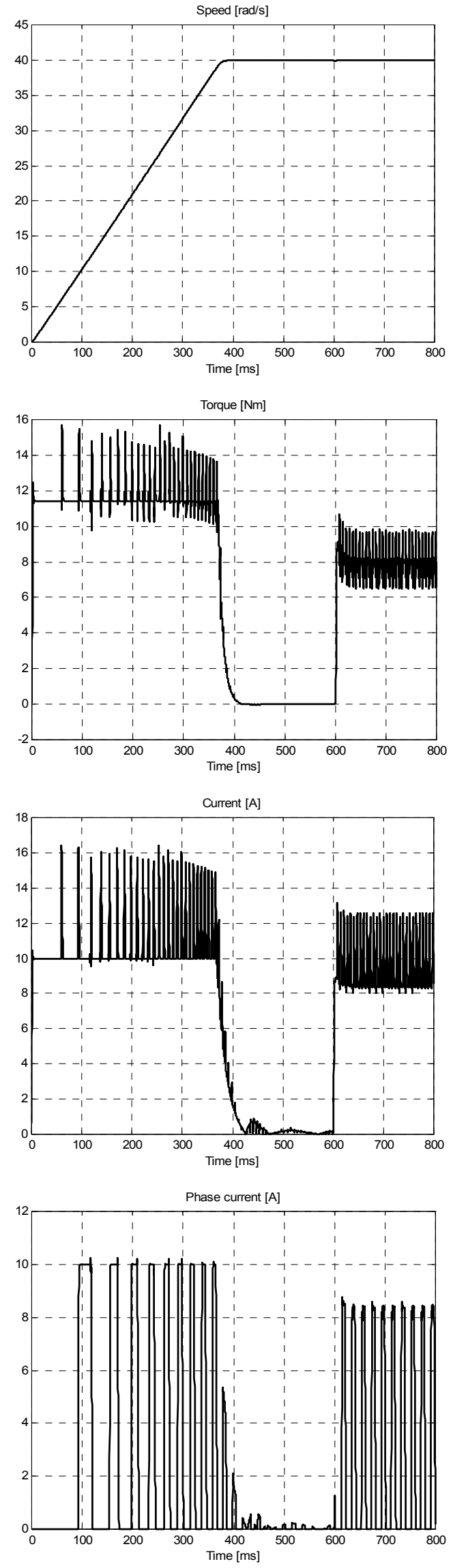

Fig. 6. Simulation results with model reference parameter adaptive speed control
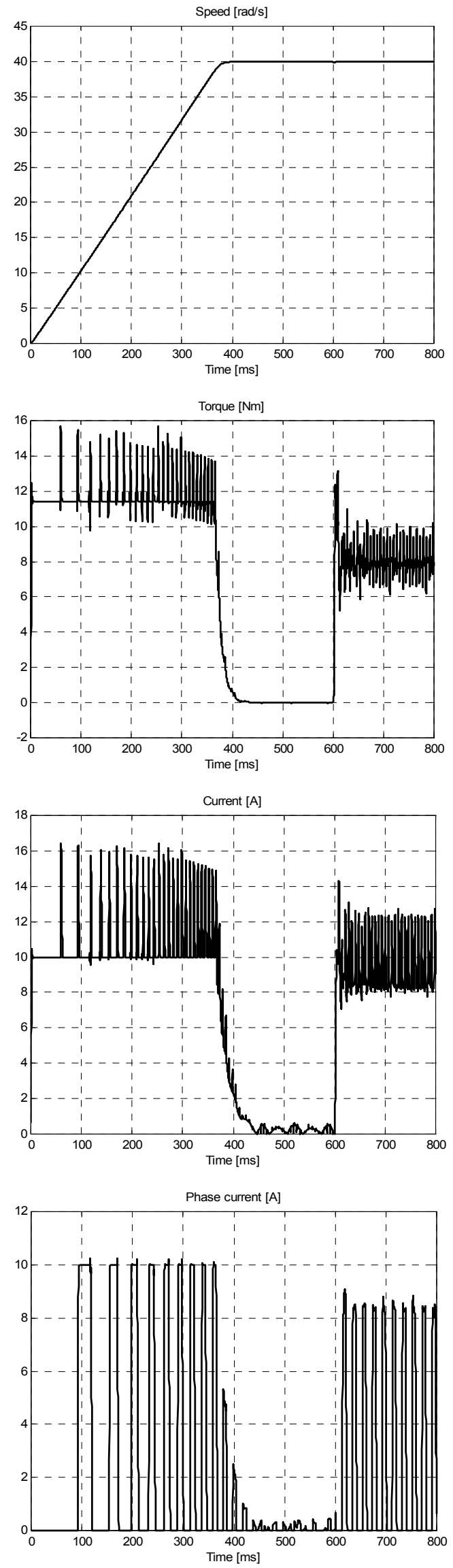

Fig. 7. Simulation results with model reference signal adaptive speed control 


\section{RESULTS}

The tests were completed by the described drive system. The test results have supported our theoretical investigations. The oscillograms in the following figures illustrate some typical starting curves and wave forms. The loading machine was a DC motor. Its inertia is about a triple of that of SRM.

Fig. 8 and Fig. 9 show the speed and current curves in the course of starting. The upper curve is the speed, the lower one is the current flowing in the common point of stator windings. It is related to the no-load operation mode.

The experiences show that the model reference parameter adaptive and signal adaptive control suggested in this paper works without overshooting.

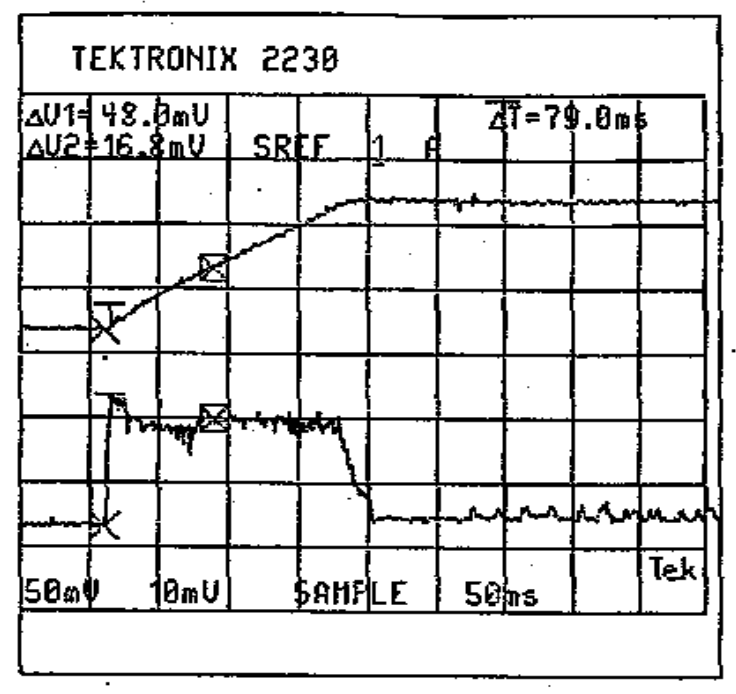

Fig. 8. Oscillogram of speed and current with model reference parameter adaptive speed control

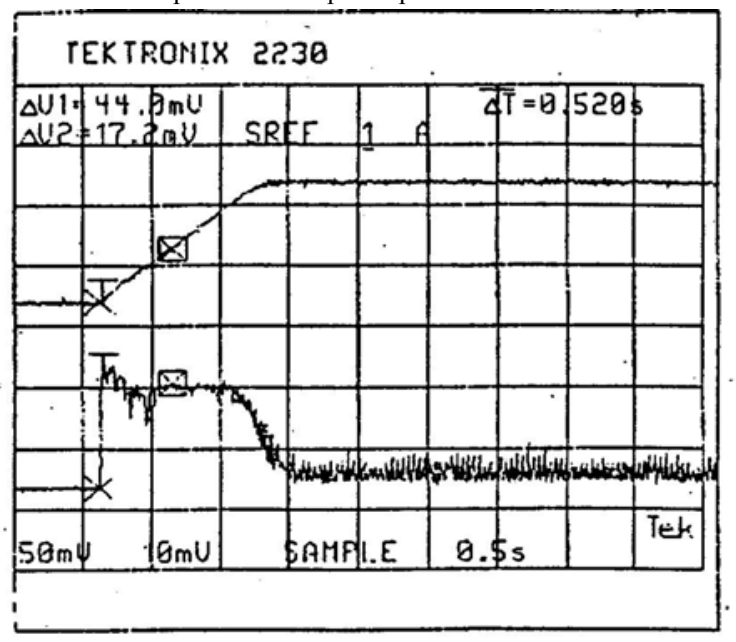

Fig. 9. Oscillograms of speed and current with model reference signal adaptive speed control

\section{CONCLUISIONS}

To provide constant loop gain in speed control loop with changing parameters (moment of inertia and/or torque factor), parameter and signal adaptive model reference adaptive control was developed.
Following from the structure (PF-type) of model reference parameter adaptive control was developed to provide constant loop gain in speed control loop with changing gain (moment of inertia and/or torque factor) it makes it easier to reach overshootless as well as fast speed changing compensation caused by jump in load. The algorithm even keeps its stability at fast changing, jumplike load torque.

Model reference signal adaptive control is used to provide constant loop gain in speed control loop with changing parameters (moment of inertia and/or torque factor) exposed to a significant load. The approaching block diagram of the adaptive control can be seen as an extended version of the PF controller, so one of the adaptation factors (which is the free parameter of the adaptive control) is given.

The adaptive controls suggested in this paper work without overshooting. Though these methods require a longer calculation period it is less sensitive to the variations of parameters. Both model reference adaptive controls drawn up can be easily implemented, because the adaptation algorithms do not need acceleration measuring (thanks to the first-order model). Simulation and experimental results demonstrate that the proposed methods are promising tools to speed control of electrical drives.

\section{REFERENCES}

[1] G. A. Perdikaris, K. W. VanPatten, "Computer Schemes for Modeling, Tuning and Control of DC Motor Drive Systems", PCI Proc., pp. 83-96, Mar. 1982.

[2] D. Y. Ohm, "A PDFF Controller for Tracking and Regulation in Motion Control", Proceedings of $18^{\text {th }}$ PCIM Conference, Intelligent Motion, Philadelphia, pp. 26-36, October 21-26, 1990.

[3] N. V. Diep, - L. Szamel, "Up-to-date Control Strategy in the Regulators of Robot Drives", PEMC'90, Budapest, Hungary, pp. 811-815, 1990.

[4] R. M. Phelan, "Automatic Control Systems", Cornell University Press, New York, 1977.

[5] Laszlo Szamel, "Model Reference Adaptive Control of SRM Drives", EPE-PEMC 2002, $10^{\text {th }}$ Int. Power Electronics and Motion Control Conf., Cavtat \& Dubrovnik (Croatia), pp. 466, CD-ROM: Paper No. T11-007, 9-11 September 2002.

[6] Laszlo Szamel, "Investigation of Model Reference Parameter Adaptive SRM Drives", EPE-PEMC 2004, $11^{\text {th }}$ Int. Power Electronics and Motion Control Conf., Riga (Latvia), 2-4, Full paper A95117 on CD_ROM, September 2004.

[7] B. K. Bose, T. G. E. Miller, "Microcomputer Control of Switched Reluctance Motor", IEEE/IAS Annual Meeting, pp. 542-547, 1985.

[8] J. Borka, K. Lupan, L. Szamel "Control Aspects of Switched Reluctance Motor Drives”, ISIE'93, IEEE International Symposium on Industrial Electronics, Budapest (Hungary), pp. 296-300 , 1-3 Juni 1993.

[9] I. Vajda, Z. Kohari, L. Benko, V. Meerovich and W. Gawalek, "Investigation of Joint Operation of a Superconducting Kinetic Energy Storage (Flywheel) and Solar Cells", IEEE Transactions on Applied Superconductivity, vol. 13, No. 2, pp. 2169-2172, June 2003.

[10] L. Szamel, "Ripple reduced control of switched reluctance motor drives", EDPE 2001, International Conference on Electrical Drives and Power Electronics, Podbanske (Slovakia), pp. 48-53, 3-5 October 2001 